\title{
Los derechos de la mujer como derechos humanos: el aporte latinoamericano a un feminismo global
}

\author{
Women's Rights as Human Rights: the Latin American \\ Contribution to Global Feminism
}

Feminism for the Americas: The Making of an International Human Rights Movement

Katherine M. Marino

The University of North Carolina Press, Chapel Hill, 2019, 354

páginas

En las historias del feminismo occidental se suele situar a la Conferencia Mundial sobre la Mujer (Beijing, 1995), organizada por las Naciones Unidas, como el foco desde el cual se propagó la idea de los "derechos de la mujer como derechos humanos" (Marino 235; Nash 91). Este evento sintetizaría la emergencia de un discurso feminista global preocupado por las cuestiones de las "mujeres del Tercer Mundo", definidas como una categoría social que suele asociarse al subdesarrollo, a las tradiciones opresivas, las altas tasas de analfabetismo, la pobreza y el fanatismo religioso (Mohanty 47). Sin embargo, investigaciones recientes han buscado disputar esta genealogía, según la cual los activismos del sur se encontrarían en desfasaje histórico o "atrasado" respecto a sus contrapartes del norte, como señalan las investigaciones de Dora Barrancos e Inés Cuadro. En esta línea se sitúa el primer libro de Katherine Marino, que busca reposicionar la producción intelectual de las feministas latinoamericanas de la 
primera mitad del siglo XX, analizando sus contribuciones dentro de una perspectiva hemisférica.

En Feminism for the Americas se analizan las tensas y complejas relaciones entre las mujeres feministas del continente en el período, cuyas discusiones dieron lugar a la defensa a nivel internacional de los derechos de las mujeres como derechos humanos fundamentales ya en la década de 1930. En su estudio, que se basa en la biografía de seis mujeres de diferentes nacionalidades, Marino reconstruye los intensos debates alrededor del feminismo en el continente, complejizando ese período que suele homogeneizarse como "primera ola" o "período sufragista”. Las vidas de Paulina Luisi (Uruguay), Bertha Lutz (Brasil), Clara González (Panamá), Ofelia Domínguez Navarro (Cuba), Doris Stevens (Estados Unidos) y Marta Vergara (Chile) estuvieron marcadas por su activismo feminista transnacional y fueron determinantes para la expansión de los derechos de las mujeres en todo el continente. Katherine Marino aporta una mirada hemisférica de los movimientos de mujeres y el impacto que tuvieron en la política internacional a partir del análisis de sus relaciones interpersonales, intercambios y tensas disputas por el poder de representación, en juego en cada uno de los congresos internacionales que se describen. Estas seis mujeres tuvieron un peso fundamental en el plano político, debido a su influencia internacional y sus privilegios como diplomáticas de sus naciones. Por su parte, las cuestiones étnico-raciales dentro y fuera de los contextos nacionales se analizan como factores que contribuyeron a la jerarquización del movimiento feminista hemisférico.

Además de las actas oficiales de los congresos, los órganos de prensa de las organizaciones feministas y artículos en los periódicos, el material de archivo con el que contó Marino abarca cartas cordiales, invitaciones a congresos, órdenes telegrafiadas, correspondencia convertida en panfletos y cartas a amantes en las que se narran confidencias y comentarios difamatorios de otras colegas. Se pueden ver las fotos personales de estas mujeres en distintos espacios de sociabilidad que ilustran un aire de época y un posicionamiento en tanto "mujeres modernas". La investigación de Marino busca reinsertar a estas seis activistas en la historiografía feminista global. Si bien es cierto que, en sus contextos nacionales, estas feministas gozan de cierto renombre, Feminism for the Americas se enfoca en sus conexiones transnacionales, sus relaciones diplomáticas y la forma en 
la que sus militancias produjeron ideas y conceptos que no les han sido suficientemente acreditados en las genealogías del feminismo global. Por este motivo, el libro es especialmente útil para pensar las relaciones a nivel hemisférico y comparativo, aun a riesgo de por momentos perder de vista ciertas especificidades de los distintos contextos nacionales. La investigación ahonda en el concepto de panamericanismo, el cual es analizado de manera cuidadosa en sus múltiples rearticulaciones y reapropiaciones por distintos sectores y agentes, con una variedad de sentidos mucho más amplia que el panamericanismo militarista de la Guerra Fría.

Los congresos y encuentros internacionales son los eventos que articulan cada uno de los capítulos y permiten a la investigadora recrear los distintos problemas y disputas que estaban en juego en cada contexto particular, dando cuenta de las alianzas y rupturas que se generaban entre feministas en cada congreso. En el primer capítulo, "A New Force in the History of the World", Marino analiza el Primer Congreso Panamericano del Niño, que tuvo lugar en la ciudad de Buenos Aires en 1916, así como los congresos en Ginebra de la Alianza Internacional para el Sufragio Femenino, y los auspiciados por la creación de la Unión Panamericana en 1910 -transformada tras la Segunda Guerra Mundial en la Organización de los Estados Americanos-, como el Congreso de Baltimore (1922), en el que surge la Asociación Panamericana para el Avance de la Mujer. Estos eventos se tradujeron en distintas organizaciones que comenzaron a ejercer una presión política cada vez mayor en los diversos gobiernos de los países americanos.

El segundo capítulo, "The Anti-imperialist Origins of International Women's Rights”, profundiza sobre las diferencias ideológicas en las distintas iteraciones del "panamericanismo", mostrando las maneras en que las feministas latinoamericanas lidiaban con el imperialismo y la creciente hegemonía de los Estados Unidos en la región, así como el uso estratégico que las feministas estadounidenses hacían de las fórmulas y el vocabulario "antiimperialista" para ganar adhesión de los latinoamericanos. Este capítulo se enfoca en las Conferencias de Panamá (1926) y de La Habana (1928), y en el rol que cumplieron Clara González, Ofelia Domínguez Navarro y Doris Stevens para la creación de una Comisión Interamericana de Mujeres. En el análisis de las intervenciones de las activistas feministas en estos congresos, Marino analiza cómo la idea de 
"derechos de la mujer", a diferencia de las sufragistas estadounidenses, ya abarcaba una forma más amplia de entender el significado de "ciudadanía", que incluía derechos sociales y económicos además de cívicos, con una agenda fuertemente marcada por las necesidades de las mujeres trabajadoras. Uno de los argumentos más interesantes del capítulo es su perspectiva sobre cómo para las feministas de los años veinte era necesario reivindicar una genealogía de ideas feministas provenientes del continente, que hacían del feminismo un movimiento vernáculo y no mera importación extranjera. Esta genealogía latinoamericana les permitía a las feministas de izquierda sostener un discurso antiimperialista al mismo tiempo que reivindicar los derechos de la mujer. El "feminismo americano” de Domínguez y González se demostró más ambicioso y radical que el de las delegadas estadounidenses en el Congreso de $\mathrm{Pa}-$ namá, quienes no veían preparadas a las latinoamericanas para ejercer el derecho de sufragio.

En este sentido, el tercer capítulo, "Feminismo práctico", analiza en detalle la recientemente creada Comisión Interamericana de Mujeres, presidida por Doris Stevens, y las tensiones y juegos de poder que se desatan dentro de la organización. A su vez, se señala cómo las jerarquías de poder manejadas por las veteranas del movimiento sufragista estadounidense también respondían a ideas de superioridad racial que aplicaban a sus compatriotas afroamericanas o indígenas. La unilateralidad con la cual Stevens manejó la presidencia de la comisión hizo que las latinoamericanas se alejaran de la asociación y buscaran rearticular sus luchas feministas por fuera de la Unión Panamericana, apelando a un panhispanismo feminista.

El cuarto capítulo, "The Great Feminist Battle of Montevideo" se centra en el Congreso de Montevideo (1933), en el cual se manifestaron explícitamente los intereses divergentes de los distintos sectores y organizaciones feministas en el hemisferio. Este congreso le permite a Marino hacer un análisis minucioso de las diferentes corrientes feministas en la región, desde las más izquierdistas hasta las más conservadoras. Ambas puntas ideológicas terminaron formando una extraña alianza, desde la brasileña Bertha Lutz y Carrie Chapman Catt y sus asociaciones de caridad, hasta Marta Vergara y sus organizaciones comunistas. En el contexto de creciente fascismo en Europa, las políticas de buena vecindad de Roosevelt y la amenaza de coartación de 
los derechos de las mujeres en el mundo, los congresos panamericanos se volvieron importantes centros de disputa ideológica. Por su parte, el antifascismo se convertiría en un núcleo aglutinador de impulsos feministas en todo el continente.

El capítulo cinco, "The Birth of Popular Front Pan-American Feminism" analiza el origen de un feminismo popular y radical que buscaba contrarrestar la hegemonía estadounidense y defender los derechos de todas las mujeres, aliándose con ideologías antirracistas, anticolonialistas y antiimperialistas. Utilizaron la agenda de "derechos iguales" para promover no solo los derechos cívicos femeninos, sino también la legislación de protección a la maternidad y a las mujeres trabajadoras. En este capítulo se perciben las tensiones entre la agenda feminista y la comunista en las activistas de la época, y cómo lograron conciliar ambos enfoques al ampliar la definición de derechos que no fueran solamente cívicos sino también sociales, económicos y políticos. El Comité Mundial de las Mujeres Contra la Guerra y el Fascismo, fundado en París en 1934, demostró ser una instancia crucial para la demarcación de una agenda feminista de frente popular que además fuese interracial. En América Latina surgió la Confederación Continental de Mujeres por la Paz (1936), que serviría como una organización alternativa y más radical que la Comisión Interamericana liderada por Stevens. Marino hace notar las diferencias de enfoque entre las feministas estadounidenses con respecto a la legislación sobre la maternidad y la manera en que muchas activistas norteamericanas señalaban las constituciones latinoamericanas como más avanzadas en la materia que la estadounidense.

En el capítulo cinco, "United Fronts for Women's Rights and Human Rights", Marino analiza cómo el contexto de la Segunda Guerra Mundial llevó a que el movimiento feminista latinoamericano tomara posturas claramente antifascistas, alineadas con el pensamiento de izquierda y la defensa de las democracias, en un período marcado por golpes de Estado apoyados por el gobierno de los Estados Unidos. El capítulo examina los eventos alrededor del Congreso Panamericano de Lima (1936), en el cual se logró sacar de su cargo a Stevens debido a la alianza entre las feministas de frente populares panamericanos y los intereses de las organizaciones gubernamentales estadounidenses. En este congreso, la mexicana Esperanza Balmaceda articula un discurso 
en el que superpone los derechos de la mujer con la idea de derechos humanos. Por este motivo, las resoluciones del Congreso de Lima son un punto clave para la articulación de los "derechos humanos" a nivel transnacional, marcando un antecedente ineludible en la conceptualización del término, ya que fue la primera vez que apareció en la resolución de un congreso de escala internacional.

El capítulo siete, "Mobilizing Women's Rights as Human Rights", se enfoca en el Congreso de Chapultepec (1945), el cual fue crucial para consolidar la relación entre derechos de la mujer y derechos humanos. Finalmente, el capítulo ocho, "The Latin American Contribution to the Constitution of the World", describe el trabajo diplomático y de presión que ejercieron las latinoamericanas en la Primera Conferencia de las Naciones Unidas (San Francisco, 1945). Estas activistas buscaron asegurar la representación de las mujeres en organismos internacionales, la creación de comisiones que supervisaran la situación de la mujer en el mundo y la inclusión de un vocabulario que explicitara la igualdad de derechos entre hombres y mujeres y la no discriminación de las personas, indistintamente de su raza, clase, sexo, nacionalidad o credo.

En suma, la investigación de Katherine Marino permite un acercamiento hemisférico al movimiento feminista en el continente en la primera mitad del siglo XX, prestando especial atención a sus manifestaciones a nivel organizativo y diplomático. El libro permite acercarse al entramado afectivo y político de los feminismos del período, y su enfoque en distintas regiones simultáneamente lo hace especialmente útil para cursos introductorios de feminismo en las Américas a nivel de grado y posgrado.

Yamile Ferreira Washington University in St. Louis, Estados Unidos http://orcid.org/0000-0002-9002-6882 yamileferreira@wustl.edu 
BibliografíA

Barrancos, Dora. Historia mínima de los feminismos en América Latina. Ciudad de México, El Colegio De México, 2020.

Cuadro Cawen, Inés. Feminismos y política en el Uruguay del Novecientos. Internacionalismo, culturas politicas e identidades de género (1906-1932). Montevideo, Ediciones de la Banda Oriental, 2018.

Marino, Katherine M. Feminism for the Americas: The Making of an International Human Rights Movement. Carolina del Norte, The University of North Carolina Press, 2019.

Mohanty, Chandra Talpade. Feminism Without Borders. Decolonizing Theory, Practicing Solidarity. Durham, Duke University Press, 2003.

Nash, Jennifer C. Black Feminism Reimagined. After Intersectionality. Durham, Duke University Press, 2019. 